\title{
Condition Based Opportunistic Maintenance Strategy of Wind Turbine
}

\author{
Gengyin LI', a, Zeyu SHAO ${ }^{2, b}$ \\ ${ }^{1}$ North China Electric Power University, China \\ ${ }^{2}$ North China Electric Power University, China \\ ashaozeyu1989@163.com, b1052364935@qq.com
}

\begin{abstract}
Keywords: wind turbine; proportional hazards model; condition indicator; maintenance threshold function

Abstract. The availability was low and the maintenance cost was expensive. In order to solve the problem, a new dynamical maintenance strategy applied to the component of wind turbine was presented, which was called condition based opportunistic maintenance strategy. In the strategy, Weibull proportional hazards model was used to describe the degradation of component. The paper used the monitoring information to defined the condition indicator which could characterize the condition of component. When and how a component should be maintained could be confirmed by comparing the value of condition indicator with the values of threshold function. The threshold function included condition maintenance threshold function and opportunistic maintenance threshold function which could be determined by minimizing the long-run expected cost rate. Finally, the practical valuableness and effectiveness of the presented strategy was verified by the the simulation analysis from economy and availability based on historical faults data and monitoring information.
\end{abstract}

\section{Introduction}

Wind power is a kind of energy with certain shortages like intermittency and instability. And on account of the complexity of design and manufacturing techniques and the harsh environment where wind turbine are usually located, unexpected failure events happen frequently. This directly leads to the expensive maintenance cost which becomes an increasingly large portion of the total operating cost of wind turbine [1]. How to reduce the cost becomes the crucial challenge to overcome for the wind turbine's managers.

For wind turbine, the positive economic dependencies between the various components actually exist [2], which here means that the cost of simultaneous maintenance of several components is lower than the sum of the costs of individual component maintenance. So with regard to the economic dependencies, opportunistic maintenance (OM) strategy was proposed and improved [2-7] to lower the total expected maintenance cost by considering the maintenance of failed components as an opportunity to maintain the additional components. Zheng and Fard [3] presented hazard rate tolerance $\mathrm{u}$ and hazard rate limit $\mathrm{L}$ to form a hazard rate interval (L-u, L) which provided a standard for preventive replacement wherein more than one components could be replaced at the same time. Zhao [6-7] presented the reliability based opportunistic maintenance strategy in which the opportunity maintenance interval was evaluated by the product of the defined opportunistic maintenance reliability margin and the reciprocal of the absolute value of slope on the reliability function. Generally, the cost rate can be minimized under the proposed OM policy like in [3, 6-7]. But the schedule of the proposed OM policy is usually made base on fixed time intervals without considering the influence of operation information that provide the diagnostic information regarding the health condition of equipment. The operation information can be detected by the condition monitoring devices which give the condition signals. In consideration of these, wind farm operators begin to realize that it is essential to make best use of the condition signals to decide when and how to perform maintenance. So, condition based maintenance (CBM) is introduced in the maintenance strategy of wind turbine. 
Proportional hazards model (PHM) [8-11] proposed by Cox [10] is a typical CBM one to model the failure pattern of components. Several papers have been published to optimize the decision making in the PHM setting. Among them, Xiang Wu et al. [11] dealt with the optimal replacement of a system having a hazard function that follows the proportional hazards model with a semi-Markovian covariate process, then the optimal threshold age was found by customizing the iterative algorithm for their model. ZHAO [8] performed CBM to main axis and main bearings of wind turbine by utilizing PHM to formulate maintenance threshold function. Therefore, the influence of operation information can be considered by PHM in making the maintenance strategy.

In this paper, we propose a new strategy implemented on wind turbine to lower the total maintenance cost. We call this strategy as condition based opportunistic maintenance (CBOM) strategy.

\section{Condition Based Opportunistic Maintenance Strategy}

Traditionally, the opportunity of OM strategy is given based on time and reliability. But CBOM proposed in this paper gives a new attempt to define the maintenance opportunity, which refers to the situation where CBM is carried out as the maintenance opportunity.

We define condition indicator (CI) to represent the operational condition, condition based maintenance threshold function (CBMTF) and opportunistic maintenance threshold function (OMTF) for each component. The CBMTF is above OMTF. While, it can be confirmed by comparing the CI with the values of CBMTF and OMTF to decide whether a component need to be maintained and how to be maintained. The detail is presented as follows. For a certain component, if its $\mathrm{CI}$ is greater than the value of $\mathrm{CBMTF}$ at a certain time, that means its operational condition deteriorates and CBM should be implemented on it, at the same time, this component's CBM give a opportunity for other components as predictive maintenance. In this occasion, if CIs of some other components are greater than their CBMTF but less than their OMTF, it means that the operational condition of these components is in close proximity to deterioration and they should implement predictive maintenance plan. This predictive maintenance for these components is defined as opportunistic maintenance, and the region between CBMTF and OMTF as condition based opportunistic maintenance region (CBOMR).

Figure 1 gives an example of three-component $(i, j, k)$ system to explain the principle of CBOM strategy. $m_{\mathrm{co}}^{(i)}(t, \boldsymbol{Z}), m_{\mathrm{co}}^{(j)}(t, \boldsymbol{Z}), m_{\mathrm{co}}^{(k)}(t, \boldsymbol{Z})$ represent the condition indicator of component $i, j$, k. $M_{\mathrm{c}}^{(i)}, M_{\mathrm{c}}^{(j)}, M_{\mathrm{c}}^{(k)}$,the CBMTF. $M_{\mathrm{o}}^{(i)}, M_{\mathrm{o}}^{(j)}, M_{\mathrm{o}}^{(k)}$, the OMTF. $\Delta M^{(i)}, \Delta M^{(j)}, \Delta M^{(k)}$, the CBOMR.

As can be seen, the maintenance actions taken on the components at $t_{1}$ include the three following cases.

-CBM action: for component $i, m_{\mathrm{co}}^{(i)}(t, \boldsymbol{Z})$ is greater than the $M_{\mathrm{c}}^{(i)}$ at $t_{1}$. CBM should be implemented on it.

-OM action: for component $j$, OM should be implemented on it because of $m_{\mathrm{co}}^{(j)}(t, \boldsymbol{Z})$ entering its $\Delta M^{(j)}$ at this very time $t_{1}$.

- No maintenance action: for component $k, m_{\mathrm{co}}^{(k)}(t, \boldsymbol{Z})$ is under the $M_{\mathrm{o}}^{(k)}$ at $t_{1}$, which means its operational condition is fine and need not to be maintained.

The final maintenance strategy at $t_{1}$ for the three-component $(i, j, k)$ system is that component $i$ and $j$ are maintained according to CBOM strategy but component $k$. This strategy can save fixed maintenance cost and decrease total downtime by maintaining two components at one time.

Consider wind turbine consisting of $N$ main components. Similarly, we define $m_{\mathrm{co}}^{(i)}(t, \boldsymbol{Z}), M_{\mathrm{c}}^{(i)}, M_{\mathrm{o}}^{(i)}, \Delta M^{(i)}$ respectively representing the CI, CBMTF, OMTF, CBOMR of component $i(i=1,2, \mathrm{~L}, N)$. The CBOM strategy for the $N$ components of wind turbine at a certain time $t$ can be described as follows. 
-CBM action should be taken for component $i(i=1,2, \mathrm{~L}, N)$ when it is satisfied over the full range of

$m_{\mathrm{co}}^{(i)}(t, \boldsymbol{Z})>M_{\mathrm{c}}^{(i)}(t)$.

- The opportunity arises once component $i$ is under CBM. OM action for component $j$ $(j \neq i \& j=1,2, \mathrm{~L}, N)$ if it is satisfied over the full range of

$$
M_{\mathrm{o}}^{(j)}(t)<m_{\mathrm{co}}^{(j)}(t, \boldsymbol{Z})<M_{\mathrm{c}}^{(j)}(t) .
$$

-No maintenance action should be taken for component $k(k \neq i \& k \neq j \& k=1,2, \mathrm{~L}, N)$ who is satisfied over the full range of

$$
m_{\mathrm{co}}^{(k)}(t, Z)<M_{\mathrm{o}}^{(k)}(t)
$$

\section{Determination of Maintenance Threshold Function Based on WPHM}

Weibull Proportional Hazards Model. Weibull proportional hazards model (WPHM) which includes methods from multivariate regression analysis into calculation of the hazard function is employed to describe deterioration process of component. Considering the effect of both age and condition information, the WPHM is expressed as

$$
h(t, \boldsymbol{Z})=h_{0}(t) \cdot \varphi(\boldsymbol{Z})=\frac{\beta}{\eta}\left(\frac{t}{\eta}\right)^{\beta-1} \cdot \exp (\gamma \cdot \boldsymbol{Z}) .
$$

Where $\boldsymbol{Z}=\left[z_{1}, z_{2}, \mathrm{~L}, z_{p}\right]$ contains $p$ condition covariates related to regression coefficient $\gamma=\left[\gamma_{1}, \gamma_{2}, \mathrm{~L}, \gamma_{p}\right] . h_{0}(t)$ is called the baseline hazards function relying on running time $t . \varphi(\boldsymbol{Z})$ is the condition covariates function only relying on condition covariates $\boldsymbol{Z}$ and reflecting the influence of $\boldsymbol{Z}$ on $h(t, \boldsymbol{Z}) . \eta$ is the life parameter. $\beta$, the shape parameter. The values of $\eta, \beta$ and $\gamma$ can be calculated using the maximum likelihood estimation algorithm according to historical fault data and condition monitoring information of each component.

Determination of Maintenance Threshold Functions and Condition Indicator. For each component, $H_{\mathrm{c}}$ is defined as the CBM threshold level. The maintenance policy is to perform CBM if the hazard function $h(t, \boldsymbol{Z})$ is greater than $H_{\mathrm{c}}$, which is shown as

$$
h(t, \boldsymbol{Z})=\frac{\beta}{\eta}\left(\frac{t}{\eta}\right)^{\beta-1} \cdot \exp (\gamma \cdot \boldsymbol{Z})>H_{\mathrm{c}} .
$$

In order to obtain the threshold functions and condition indicator, (3) is made the following changes turning into equation (4).

$$
\gamma \cdot \boldsymbol{Z}>\ln \left(\frac{\eta^{\beta} H_{\mathrm{c}}}{\beta}\right)-(\beta-1) \ln t .
$$

Obviously, the right side of (4) is a function of $t$, and the left side is a "combined covariate value" reflecting the operational condition. It has the same expression with equation (1a). So, we make the following definitions for each side of equation (4) as equation (5) and equation (6)

$$
m_{\mathrm{co}}(t, \boldsymbol{Z}) @ \gamma \cdot \boldsymbol{Z} .
$$




$$
M_{\mathrm{c}}(t) @ \ln \left(\frac{\eta^{\beta} H_{\mathrm{c}}}{\beta}\right)-(\beta-1) \ln t
$$

Where $m_{\mathrm{co}}(t, \boldsymbol{Z})$ is defined as CI which is a weighted sum of condition covariates. $M_{\mathrm{c}}(t)$ is defined as CBMTF which is a function only depending on $t$.

Similarly, OMTF can be defined as the following form,

$$
M_{\mathrm{o}}(t) @ \ln \left(\frac{\eta^{\beta} H_{\mathrm{o}}}{\beta}\right)-(\beta-1) \ln t .
$$

Where $H_{\mathrm{o}}$ is defined as $\mathrm{OM}$ threshold level of the component.

For the $N$ main components of wind turbine, the $M_{\mathrm{c}}^{(i)}(t)$ and $M_{\mathrm{o}}^{(i)}(t)$ of component $i$ $(i=1,2, \mathrm{~L}, N)$ can be defined as

$$
\begin{aligned}
& M_{\mathrm{c}}^{(i)}(t)=\ln \left(\frac{\eta^{(i)^{\beta^{(i)}}} H_{\mathrm{c}}^{(i)}}{\beta^{(j)}}\right)-\left(\beta^{(i)}-1\right) \ln t . \\
& M_{\mathrm{o}}^{(i)}(t)=\ln \left(\frac{\eta^{(i)^{\beta^{(i)}}} H_{\mathrm{o}}^{(i)}}{\beta^{(j)}}\right)-\left(\beta^{(i)}-1\right) \ln t .
\end{aligned}
$$

$H_{\mathrm{c}}^{(i)}$ and $H_{\mathrm{o}}^{(i)}$ in equation (8) and equation (9) satisfy equation (10) and equation (11).

$$
\begin{aligned}
& H_{\mathrm{c}}^{(i)}=\frac{\beta^{(i)}}{\eta^{(i)}}\left(\frac{T_{\mathrm{c}}^{(i)}}{\eta^{(i)}}\right)^{\beta^{(i)}-1} \cdot \exp \left(\gamma^{(i)} \cdot \boldsymbol{Z}_{\mathrm{c}}^{(i)}\right) . \\
& H_{\mathrm{o}}^{(i)}=\frac{\beta^{(i)}}{\eta^{(i)}}\left(\frac{T_{\mathrm{o}}^{(i)}}{\eta^{(i)}}\right)^{\beta^{(i)}-1} \cdot \exp \left(\gamma^{(i)} \cdot \boldsymbol{Z}_{\mathrm{o}}^{(i)}\right) .
\end{aligned}
$$

To calculate $M_{\mathrm{c}}^{(i)}(t)$ and $M_{\mathrm{o}}^{(i)}(t), H_{\mathrm{c}}^{(i)}$ and $H_{\mathrm{o}}^{(i)}$ should be verified. $H_{\mathrm{c}}^{(i)}$ and $H_{\mathrm{o}}^{(i)}$ can be calculated by utilizing the optimal CBM time $T_{\mathrm{c}}^{(i)}$ and the optimal OM time $T_{\mathrm{o}}^{(i)}$.

After obtaining the $M_{\mathrm{c}}^{(i)}(t)$ and $M_{\mathrm{o}}^{(i)}(t)$, the dynamic maintenance process is assured as the flow chart in figure 2 .

\section{The Verification of CBMTF and OMTF}

The method of minimizing the long-run expected cost rate is adopted to calculate $T_{\mathrm{c}}^{(i)}$ and $T_{\mathrm{o}}^{(i)}$. As is seen

$$
c(T)=\frac{\text { Total expected cost per cycle }}{\text { Expected cycle length }} \text {. }
$$

In which, a optimal maintenance time $T$ can be calculated. This paper assumes the following basic decision rule: if no failures occur until the $T, \mathrm{CBM}$ should be performed on the component immediately; but the component receives different maintenance action if suffering a sudden failure before $T$. With different maintenance action, the description of equation (12) is different, so is $T$. 
For calculating CBMTF, this paper suggests overhaul after sudden failure before $T$. Whether overhaul or CBM makes the component as new. Then (12) can be described as

$$
c(T)=\frac{\left(C_{\mathrm{F}}+C_{\mathrm{d}}+C_{f}\right) F(T)+\left(C_{\mathrm{c}}+C_{\mathrm{d}}+C_{f}\right) R(x)}{\int_{0}^{T} R(x) d x} .
$$

Where $R(x)$ is the reliability function, and $F(T)$ the failure distribution function. $C_{\mathrm{F}}$ is the overhaul cost, $C_{\mathrm{d}}$ the downtime cost, $C_{\mathrm{c}}$ the CBM cost. and $C_{f}$ the fixed maintenance cost which contains manual cost, tools cost and crane cost etc accounting for a large part of total maintenance cost and arises every time when maintenance is performed.

But for calculating OMTF, minor repair is suggested after sudden failure before $T$. It does not affect the proportional hazards function of component. The component keeps running after minor repair. So the long-run expected cost rate is

$$
c(T)=\frac{\left(C_{\mathrm{o}}+C_{\mathrm{d}}+C_{f}\right)+C_{\mathrm{m}} \int_{0}^{T} h(x, \boldsymbol{Z}(x)) d x}{T} .
$$

Where $h(x, \boldsymbol{Z}(x))$ is the hazards function. $C_{\mathrm{o}}$ is the OM cost, $C_{\mathrm{m}}$ the minor repair cost. $C_{\mathrm{d}}$.

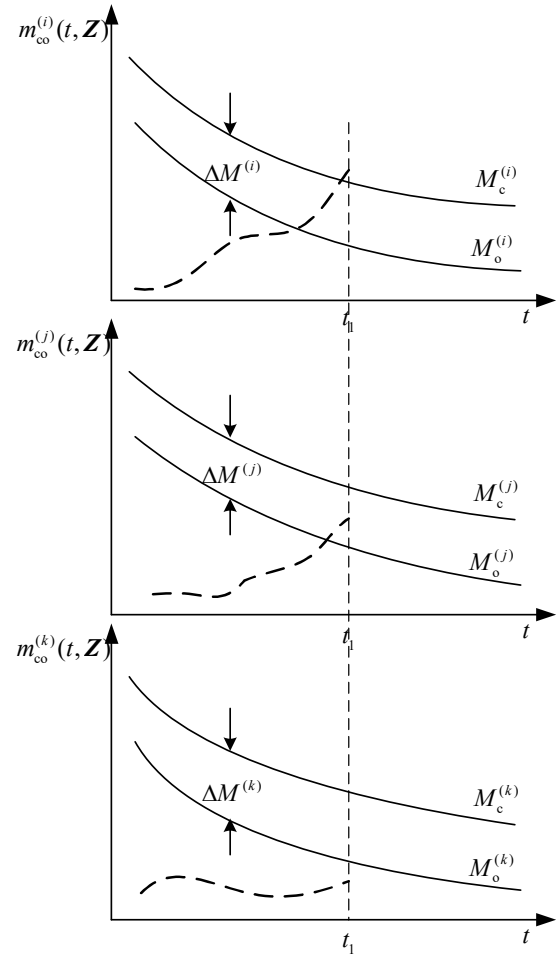

Figure.1 Diagram of CBOM Strategy

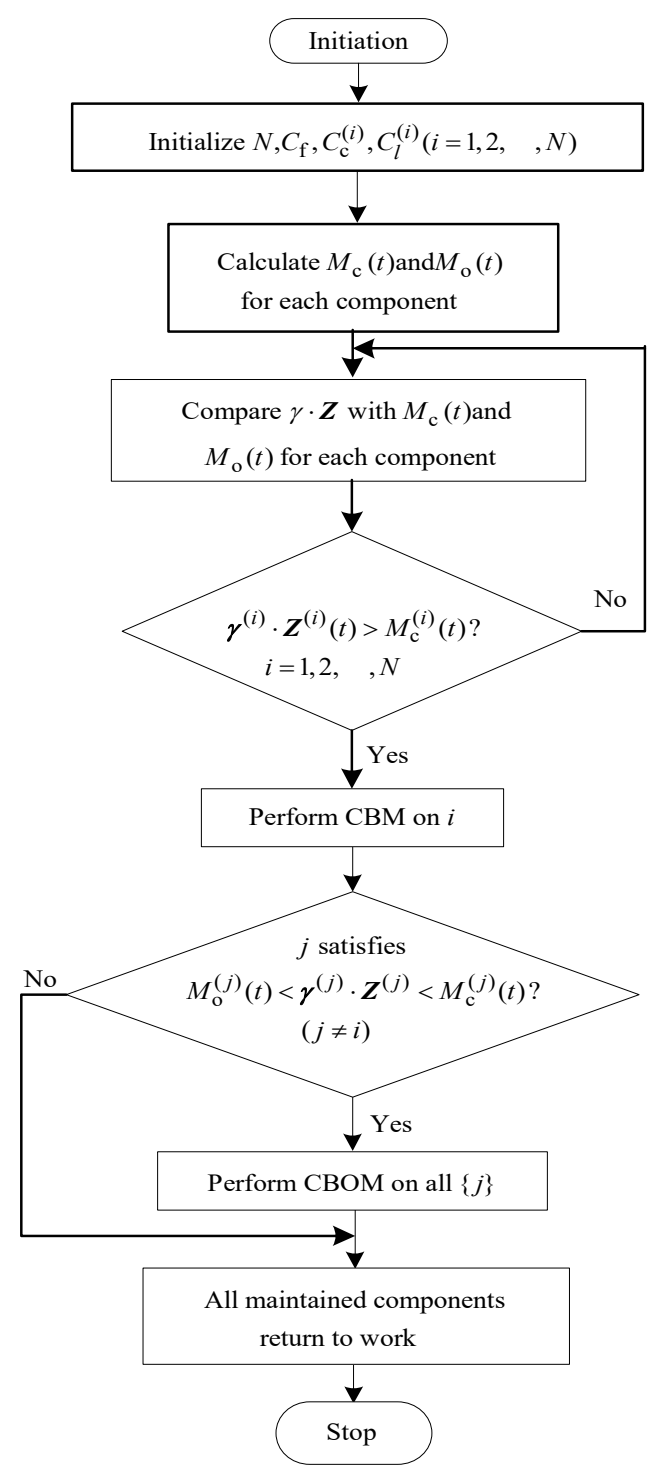

Figure.2. The Flow Chart of Maintenance Action 
The optimal CBM time $T_{\mathrm{c}}$ and the optimal OM time $T_{\mathrm{o}}$ of each component can be calculated by minimizing equation (13) and equation (14) respectively. Then $M_{\mathrm{c}}(t)$ and $M_{\mathrm{o}}(t)$ of each component can be determined.

\section{Cost Analysis of CBOM Strategy}

The total maintenance cost at each maintenance point can be denoted as the algebraic sum of CBM cost, the downtime cost and fixed maintenance cost. This paper defines set $A_{\mathrm{c}}^{(i)}$ and $\operatorname{set} A_{\mathrm{o}}^{(i)}$ to show the CBM action and OM action for component $i$ :

$$
\begin{aligned}
& A_{\mathrm{c}}^{(i)}=\left\{\begin{array}{ll}
1, & \mathrm{CBM} \text { for component } i \\
0 & \text { No maintenance action }
\end{array} .\right. \\
& A_{\mathrm{o}}^{(i)}=\left\{\begin{array}{ll}
1, & \text { OM for component } i \\
0 & \text { No maintenance action }
\end{array} .\right.
\end{aligned}
$$

So the form of total maintenance $\operatorname{cost} C_{c o-t o t a l}$ is showed below.

$$
C_{c o-t o t a l}=\sum_{i=1}^{N} A_{\mathrm{c}}^{(i)} \cdot\left(C_{\mathrm{c}}^{(i)}+C_{0}\right)+\sum_{j=1}^{N} A_{\mathrm{o}}^{(j)} \cdot C_{\mathrm{o}}^{(j)}+C_{l}^{(q)} .
$$

Where component $i$ is the one under CBM and $j$ under COBM. The $q$ is the one that has the maximum the downtime cost among all the components under maintenance.

If CBM is performed for single component without considering $\mathrm{OM}$, the total $\operatorname{cost} C_{c-t o t a l}$ can be obtained as

$$
C_{c-t o t a l}=\sum_{i=1}^{N} A_{\mathrm{c}}^{(i)} \cdot\left(C_{\mathrm{c}}^{(i)}+C_{l}^{(i)}+C_{0}\right)
$$

\section{Analysis of Examples}

Calculation of Proportional Hazards Function for Each Component. Four key components from one kind of wind turbine is chosen as the example for the analysis of CBOM strategy. And we number the four components from 1 to 4 . The historical faults data are shown in Table 1 . And the vibration velocity (V-V) monitoring data/millimeter varying with time(day) are shown in Table 2

Table 1. Historical Faults Data of Each Component

\begin{tabular}{cccccccccccc}
\hline Component 1(day) & 130 & 126 & 1147 & 153 & 142 & 130 & 157 & 162 & 164 & 172 & 179 \\
& 4 & 8 & 1 & 2 & 4 & 9 & 1 & 0 & 3 & 0 \\
Component 2(day) & 120 & 106 & 134 & 143 & 122 & 160 & 147 & 162 & 164 & 152 & 1190 \\
& 4 & 8 & 7 & 1 & 2 & 4 & 9 & 1 & 0 & 3 & \\
Component 3(day) & 915 & 127 & 136 & 1151 & 153 & 142 & 149 & 155 & 164 & 168 & 163 \\
& & 9 & 4 & & 4 & 9 & 0 & 8 & 9 & 8 \\
Component 4(day) & \multirow{2}{*}{856} & 106 & 955 & 142 & 122 & 1131 & 139 & 164 & 100 & 1145 & 129 \\
& & 8 & & 8 & 2 & & 7 & 6 & 3 & & 8 \\
\hline
\end{tabular}


Table 2. Vibration Signal Monitoring Data of Each Component

\begin{tabular}{cccccccc}
\hline \multicolumn{2}{c}{ Component 1 } & \multicolumn{2}{c}{ Component 2 } & \multicolumn{2}{c}{ Component 3 } & \multicolumn{2}{c}{ Component 4 } \\
\hline Time(day) & $\begin{array}{c}\mathrm{V}-\mathrm{V} \\
(\mathrm{mm} / \mathrm{s})\end{array}$ & Time(day) & $\begin{array}{c}\mathrm{V}-\mathrm{V} \\
(\mathrm{mm} / \mathrm{s})\end{array}$ & Time(day) & $\begin{array}{c}\mathrm{V}-\mathrm{V} \\
(\mathrm{mm} / \mathrm{s})\end{array}$ & Time(day) & $\begin{array}{c}\mathrm{V}-\mathrm{V} \\
(\mathrm{mm} / \mathrm{s})\end{array}$ \\
\hline 21 & 6.730 & 22 & 1.422 & 23 & 5.488 & 25 & 1.641 \\
135 & 6.789 & 137 & 1.471 & 132 & 5.533 & 146 & 1.711 \\
348 & 6.801 & 364 & 1.511 & 265 & 5.986 & 162 & 1.723 \\
561 & 6.819 & 586 & 1.542 & 497 & 5.614 & 284 & 1.743 \\
679 & 6.865 & 607 & 1.598 & 618 & 5.922 & 307 & 1.804 \\
782 & 7.904 & 824 & 1.653 & 837 & 6.645 & 431 & 1.851 \\
891 & 8.984 & 1042 & 1.768 & 968 & 7.135 & 552 & 1.989 \\
1000 & 9.432 & 1151 & 2.298 & 1017 & 7.778 & 674 & 2.022 \\
1121 & 9.987 & 1160 & 4.361 & 1168 & 10.397 & 803 & 2.657 \\
1139 & 10.783 & 1269 & 7.923 & 1190 & 12.481 & 926 & 3.997 \\
1152 & 16.115 & & & 1217 & 24.875 & 935 & 6.876 \\
1178 & 25.321 & & & 1238 & 28.875 & 1044 & 8.745 \\
& & & & & & 1053 & 12.113 \\
\hline
\end{tabular}

The WPHM parameters of each component are calculated and shown in Table 3.

Determination of CBOMR for Each Components. All kinds of maintenance cost is shown in Table 4. $H_{\mathrm{c}}^{(i)}$ and $H_{\mathrm{o}}^{(i)}$ can be obtained according to the data in Table 4 and shown in Table 5. Then the CBMTF and OMTF shown in figure 3. can be determined.

Table 3. Values of WPHM Parameters

\begin{tabular}{|c|c|c|c|c|}
\hline $\begin{array}{c}\text { Component } \\
\text { No. }\end{array}$ & 1 & 2 & 3 & 4 \\
\hline$\beta$ & $\begin{array}{c}7.836 \\
6\end{array}$ & $\begin{array}{c}8.573 \\
6\end{array}$ & $\begin{array}{c}9.183 \\
6\end{array}$ & 5.6468 \\
\hline$\eta$ & $\begin{array}{c}1377 . \\
6\end{array}$ & $\begin{array}{c}1485 . \\
5\end{array}$ & $\begin{array}{c}1392 . \\
6\end{array}$ & 1288.5 \\
\hline$\gamma$ & $\begin{array}{c}0.315 \\
5\end{array}$ & $\begin{array}{c}0.857 \\
6\end{array}$ & $\begin{array}{c}0.186 \\
4\end{array}$ & 0.6760 \\
\hline
\end{tabular}

Table 4. Maintenance Cost of Each Component

\begin{tabular}{ccccc}
\hline Component No. & 1 & 2 & 3 & 4 \\
\hline$C_{\mathrm{F}}(¥)$ & 12.0 & 10.3 & $\begin{array}{c}18 . \\
8\end{array}$ & 14.7 \\
$C_{\mathrm{m}}(¥)$ & 6.0 & 7.7 & 8.6 & 7.5 \\
$C_{\mathrm{c}}(¥)$ & 11.2 & 8.4 & 15. & 12.6 \\
$C_{l}(Y)$ & 7.4 & 6.8 & 9.2 & 8.6 \\
$C_{f}(Y)$ & & & 8.0 & \\
\hline
\end{tabular}

Table 5. $H_{\mathrm{c}}^{(i)}$ and $H_{\mathrm{o}}^{(i)}$ of Each Component

\begin{tabular}{ccccc}
\hline $\begin{array}{c}\text { Componen } \\
\mathrm{t} \text { No. }\end{array}$ & 1 & 2 & 3 & 4 \\
\hline$H_{\mathrm{c}}^{(i)}$ & 0.1687 & 1.2095 & 0.0110 & 0.5504 \\
$H_{\mathrm{o}}^{(i)}$ & 0.0138 & 0.0470 & 0.0014 & 0.1151 \\
\hline
\end{tabular}



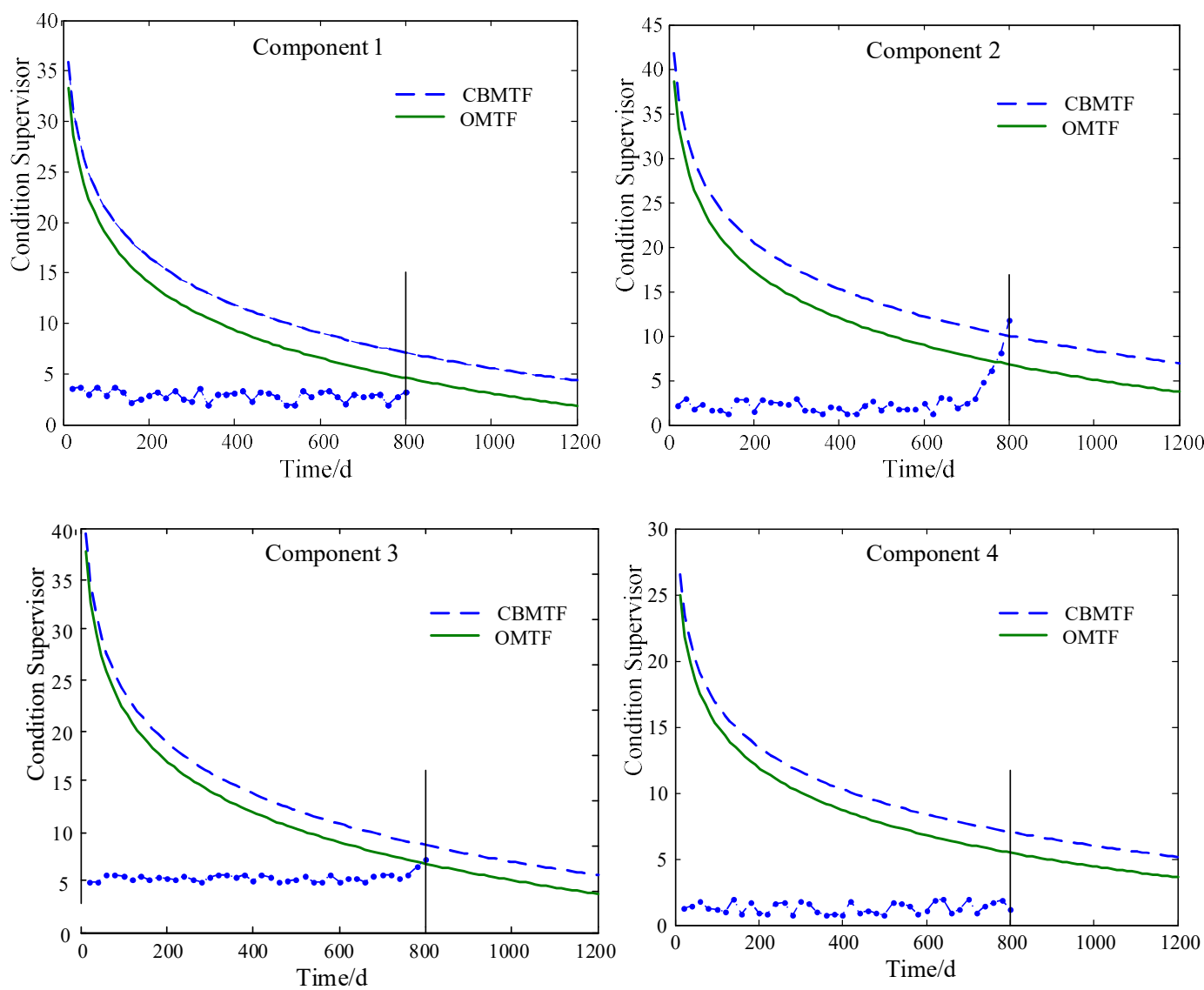

Figure 3. The Flow Chart of Maintenance Action

Computing Analysis of CBOMR Strategy. It can be seen from figure 3 that the CI of Component. 2 is greater than the value of its CBMTF and The CI of Component. 3 entered its CBOMR. Then we perform CBM on Component.2 and implement CBOM on Component.3. But for Component.1 and 4, their CIs are less than the value of its OMTF, which means the operational condition is fine and they do not need any maintenance. Considering the cost in Table 4, we can get the total cost, 415 thousands Yuan, by adopting COBM and using equation (17). If we insist on performing CBM on Component. 3 after its $\mathrm{CI}$ is beyond the CBMTF, it can be calculated by equation (18) that the total cost will be up to 563 thousands Yuan. In addition, only one fixed maintenance cost and one downtime cost arise in this maintenance, which saves the total maintenance cost. After maintenance, In comparison, CBOM saves 148 thousands Yuan less than CBM for single component. The saving rate is up to $26.3 \%$, which verifies the effectiveness of $\mathrm{CBOM}$ in wind turbine maintenance.

\section{Conclusions}

This paper proposed a condition based opportunistic maintenance strategy whose threshold level is a dynamical threshold function for wind turbine. This strategy can save the total maintenance cost and reduce downtime effectively. It can provide theoretical foundation for the operation and maintenance of wind turbine. But in this paper, we just considered one condition covariate, in the next work, we will consider more.

\section{References}

[1] YAN Sheng-teng Optimization Maintenance of Wind Turbine Based on Opportunistic Maintenance Model (2012) 
[2] Sethi, D.P.S. Opportunistic Replacement Policies, in: Tsokos, C.P. and Shimi, I.N. (Ed), The Theory and Application of Reliability, 1, p. 433-47, Academic Press, New York(1997)

[3] Zheng X, Fard N A maintenance policy for repairable systems based on opportunistic failure rate tolerance IEEE Transactions on Reliability,40 ,p 237-44(1991)

[4] Nilsson J. 2009 An opportunistic maintenance optimization model for shaft seals in feed-water pump systems in nuclear power plants IEEE Bucharest Power Technology, Bucharest Romania(2009)

[5] Francois Besnard An optimization framework for opportunistic maintenance of offshore wind power system. IEEE Bucharest Power Technology Conf Bucharest Romania(2009)

[6] Zhao Hongshan, Yan Shengteng, Zhang Xiaotian Deterministic opportunistic maintenance strategy for wind turbine Acta Energiae Solaris Sinica, 35(4) p 567-75(2014)

[7] Zhao Hongshan, Zhang, Lupeng Preventive Opportunistic Maintenance Strategy for Wind Turbines Based on Reliability Proceedings of the CSEE, 34(22) p 3777-83(2014)

[8] ZHANG Ji-quan Optimized Maintenance Decision of wind turbine-generator system based on proportional intensity model (2010)

[9] Xiang Wu and Sarah M. Ryan Optimal Replacement in the Proportional Hazards Model With Semi-Monrovian Covariate Process and Continuous Monitoring. IEEE Transactions on Reliability (2011)

[10]Cox, D.R. and Oakes, D. 1984 Analysis of Survival Data, Chapman \& Hall, London(1984) 